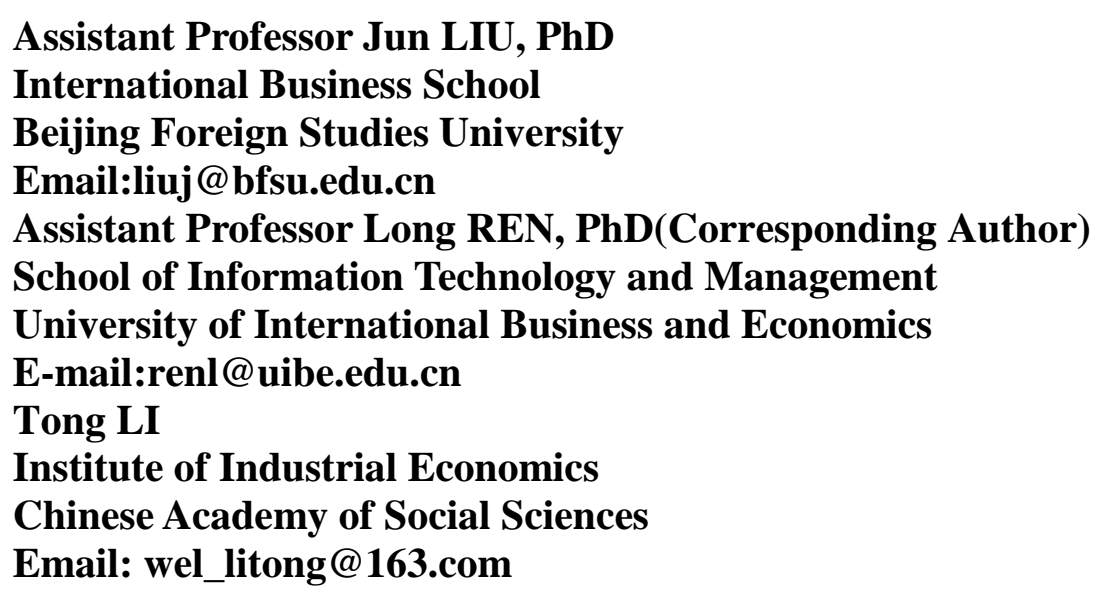

\title{
HOW MANY ROBOTS SHOULD WE INVEST? A THEORETICAL ANALYSIS WITH TWO-SECTOR PRODUCTION FACILITY
}

\begin{abstract}
With the ascending of AI, robots have become important production factors and have been put into production on a large scale. In this paper, we build up a two-sector production model considering the robots investments, in which the manufacturer decides how many robots to invest. We find that the optimal robots quantity is increasing in the capital. It is decreasing in the variable costs and concave in the quantity of labour who can be replaced by robots easily. Further, we analyse the impacts of robots investments on workforce wages. As the number of robots increases, the wage in the tradition production sector increases while the wage in the production sector with robots decreases, which means that the wage gap between these two sectors deepens. We provide more managerial insights not only from the perspective of the two-sector manufacturer but also from the point of view of a social planner.

Keyword: Production, Robots, Technology innovation, Artificial intelligence, Wage gap.
\end{abstract}

JEL classification: D24, J31, O33

DOI: $10.24818 / 18423264 / 54.2 .20 .12$ 


\section{Introduction}

Recent years have witnessed a rapid growth of the demand and sales of robots globally. According to relevant data provided by the International Robot Alliance (IFR), the global demand for robots is expected to grow at a rate of $12 \%$ per year from 2016 to 2019, and the total global robot sales project to reach 410,000 units by 2019. Therefore, the development trends of the robot market cannot be underestimated.

With the ongoing development of artificial intelligence and machine learning technology, the use of robots is also spreading. In July 2016, McKinsey \& Company released a report titled "Which fields will be replaced by machines, and which will not." The article argues that as machine learning continues to advance, many jobs, especially the manual labour, are the most likely to be replaced. In addition, with the emergence of driverless technology, robot investment adviser, electronic medical equipment and distribution drones, drivers, investment consultants, couriers, doctors and other professions that are closely related to people's lives will also suffer the impacts brought by robots. At present, the use of robots has replaced some labour force in the production process. Empirical studies have shown that the use of robots does reduce the share of labor (AcemogluandRestrepo, 2017; AutorandSalomons, 2018). According to one report from CNBC (Consumer News and Business Channel) on October 27, 2017, retail giant Wal-Mart has deployed rack-scanning robots in more than 50 locations in the United States, to check inventory, prices and misplaced items. Thus, it can be seen that artificial intelligence and robots are being applied to production and daily life on a large scale. With the increasing use of robots and the continuous development of the world robot market, it is bound to have a profound impact on economic growth. Existing empirical studies support the view that the use of industrial robots will increase labour productivity (Kromann et al., 2011; GreatzandMicheals, 2018). Robots are inevitably changing our lives, but in what form and to what extent will they change people's lives? Because of the increasing cost of traditional capitals, such as oil (Su et al, 2017,2019) and the fierce competition between online and offline businesses (Gong et al, 2019), enterprises seeks to take advantage of the high efficiency and economic benefits of robots in the production process nowadays. In exchange, they have to pay for the high costs of robots. So, our first question is the more robots invested in the production process, the better? Furthermore, while people enjoy the convenience brought by robots, they are also exposed to the employment challenges that robots will gradually replace the labor work. So, our second question- what the influences of large-scale application of robots on the wages of labor- is also worthy of exploring.

Motivated by the above analysis, this paper mainly answers the following two research questions: 1) From the perspective a manufacturer, taking robots into the production process, what the relationship between the profit of the manufacturer and the quantity of the robot invested, whether there is an optimal investment level, and what are the driven forces behind; 2) From the perspective of labor forces, with the development of artificial intelligence, how the ascending of robots will affect

DOI: $10.24818 / 18423264 / 54.2 .20 .12$ 
How Many Robots Should We Invest? A Theoretical Analysis with Two-Sector Production Facility

the labor market, especially how the labor wages will change? At present, robots can almost completely replace the labor force in some industries. For examples, in the welding workshop of the automotive industry and the manual processing workshop in the metal industry, robots play more important roles in the production process. The utilization of robots to diagnose diseases, translate words, write news and reports is becoming more popular in other fields, which gradually reduce the reliance on the human labor. However, not all work types can be perfectly replaced by robots. Frey and Osborne (2017) summarized three categories of tasks that are not easily taken places by robots: perception and manipulation tasks, creative tasks and social intelligence tasks. This paper argues that we cannot generalize the substitution effects of robots to all industries. However, the substitution effects should be discussed in different departments in the current production process of enterprises.

In order to answer the above research questions, we establish a two-sector production model by taking robots into the production process. Through the analysis of the model, this study finds that the optimal number of robot inputs is increasing in the quantity of capital, decreasing in variable cost, and concave in the number of labor that can be replaced by robots. As the number of robots invested increases, the wages of the traditional production sector will increase, while the wages of the robotics sector will decrease, deepening the social wage gap and bringing more social problems.

The structure of this paper is as follows. Section 2 reviews relevant literature, mainly from the streams of robots in the production function, the impact of robots on the wages of human capital and economic development. Section 3 establishes a two-sector production model with the consideration of robots. Section 4 investigates the relationship between the profit of the manufacturer and the investment level of robots and identifies the driven forces of the optimal investment level. Section 4analyses the relation between the wage gap of human capital and the robot investment level. Towards the end of this paper, we illustrate the theoretical results more intuitively through numerical studies in Section 5. Finally, we draw conclusions in the last section of this paper.

\section{Literature review}

As for the literatures considering how to take the robots into production function, Acemoglu and Autor (2011) propose the Task-based Model. This model can be traced back to Zeira (1998), whose paper expresses the final output as an integral form of some intermediate inputs. Acemoglu and Restrepo (2018) extended the model, assuming that the final product consists of continuous tasks, and each task is completed by both robots and human capital. Specifically, there is a unique final product $Y$ in the economy, which is produced by a continuous task $y(i)$. The specific production function form is 
Jun Liu, Long Ren, Tong Li

$Y=\left(\int_{N-1}^{N} y(i)^{\frac{\sigma-1}{\sigma}} d i\right)^{\frac{\sigma-1}{\sigma}}$,

(1)

where $\sigma \in(0, \infty)$ is the substitution elasticity. Prettner (2017) modified the Solow model (1956) using the Cobb-Douglas production function form. They divided the capital part of the production function into traditional capitals and robots, such as 3D printers, driverless cars, etc. Then they introduced them into the Solow model. The specific production function form is

$Y(t)=A(t)[L(t)+P(t)]^{1-\alpha} K(t)^{\alpha}$,

where $Y(t)$ represents the total output, $A(t)$ represents the technology development level, $L(t)$ represents labor, $P(t)$ represents the automated capital (robots), and $K(t)$ is the traditional capital. Graetz and Michaels (2018) established a theoretical framework for robots that divided the production process into two sectors: one consists of robots and human capital, and the other consists of only human capital. By assuming that the consumer's utility function is with constant elasticity and the consumption comes from the total output, the consumer's utility function can be expressed as

$U=\left(Y_{R}^{\frac{\varepsilon-1}{\varepsilon}}+Y_{N}^{\frac{\varepsilon-1}{\varepsilon}}\right)^{\frac{\varepsilon}{\varepsilon-1}}$,

(3)

where $Y_{R}$ is the department that uses the robot and human capital, and $Y_{N}$ is the department that only uses human capital. Benzell et al. (2015) represented the smart machine as a combination of capital and code. They divided the labor in the labor market into high-tech and low-tech labor, where high-tech labor can produce code. By establishing a two-period overlapping generation model and using numerical simulations, it is demonstrated that the participation of robots in the long term will reduce the wages of the labour force.

At present, there have been some empirical studies that prove that technological progress will increase the wage gap, thus causing the widening social gap (Tinbergen, 1974, 1975;Katz and Murphy, 1992;Autor et al., 1998;Carneiro and Lee, 2009;Acemogluand Autor, 2011), but these studies only focus on the impact of technological progress on the wage gap. As for related research on the influence of the use of robots in labour wages and economic development, Sachs and Kotlikoff (2012) argued that robots are a substitute for unskilled young labourers and a complement to highly skilled labourers. Thus, the use of robots will reduce the wages of the younger generation. Prettner and Strulik (2017) applied the OLG model and introduced educational decision and fertility decision into the model. The model assumes that in the production process, people with high education are complementary to machines and those with low education are substitutes, which proves that the continuous development of the economy brings about a further increase in the income gap. Gasteiger and Prettner (2017) used the Diamond (1965) model to conclude that the use of robots ultimately led to economic stagnation, as the use of robots inhibited wage growth and thus inhibited investment growth.

DOI: $10.24818 / 18423264 / 54.2 .20 .12$ 
How Many Robots Should We Invest? A Theoretical Analysis with Two-Sector Production Facility

To summarize, we find that: 1) Little literature studies the impact of the employment of robots on enterprise profits from the perspective of production; 2) Little literature such as Graetz and Michaels (2018) investigate the effects of robots when different production sectors exist. Specifically, Graetz and Michaels (2018) divided the production process into two divisions: one consisting of robots and human capital, and the other consisting of only human capital. However, they did not consider the role of traditional capital in production, and not analyze and discuss the micro level of labor market wages and corporate profits as well. In contrast, this paper establishes a more general model of a two-sector production function considering the investments of robots and its substitution effects, based on which we conduct further analysis.

\section{Model Setup}

The basic assumptions of the two departments in this paper consider the following situations in daily-life: the office lobby of the bank are divided into two categories, counter labor service and ATM self-service; the supermarket cashiers counter are divided into two categories, manual cash register and machine automatic cash register. There are also two types of departments in some factories. One department can use robots and other capitals at the same time, while other departments rely on traditional human and capital inputs, and these two types of production departments are usually substituted in some degrees. Therefore, this paper assumes that the production enterprise can be divided into two departments, namely, the traditional production sector (the production department without robots' participation) and the production sector with robots. In the traditional production sector, robots cannot replace human labor. $Y_{T}$ indicates the output of the traditional production sector. $K_{T}$ denotes the capital used in the traditional sector, and $L_{T}$ indicates the labor used in the traditional sector, where the labor is not easily replaced by robots. We assume that the production function follows a Cobb-Douglas form, where $\beta$ is the elasticity coefficient. To that end, the total output of the traditional production sector is

$Y_{T}=L_{T}^{1-\beta} K_{T}^{\beta}$.

(4)

In addition to the traditional sector, there is a substituting department in the enterprise. $Y_{R}$ indicates the output of that department. $K_{R}$ indicates capital used in the sector with robots. $L_{R}$ indicates the human labor existing in the department, and $\alpha$ is the coefficient of elasticity of the department, where labor can be replaced by the robot. The product production function that can be replaced by the robot production department is

$Y_{R}=\left(L_{R}+R\right)^{1-\alpha} K_{R}^{\alpha}$.

(5)

Let $\theta$ and $1-\theta$ indicate the intensive parameters of the traditional sector and the robotic alternative sector, respectively. $\sigma$ indicates the alternative elasticity of

DOI: 10.24818/18423264/54.2.20.12 
Jun Liu, Long Ren, Tong Li

the production function. The total production function of the constant substitution elasticity is

$Y=\left(\theta Y_{T}^{\frac{\sigma-1}{\sigma}}+(1-\theta) Y_{R}^{\frac{\sigma-1}{\sigma}}\right)^{\frac{\sigma}{\sigma-1}}$.

(6)

When $\sigma \rightarrow \infty, Y=\theta Y_{T}+(1-\theta) Y_{R} ; \sigma \rightarrow 1, Y=Y_{R}{ }^{\theta} Y_{T}{ }^{1-\theta}$.

Therefore, the total production function satisfies the Cobb-Douglas form.

The cost elements of the enterprise are summarized in Table 1:

Table 1. Cost Elements

\begin{tabular}{cc}
\hline Notations & Meanings \\
\hline $\boldsymbol{w}_{\boldsymbol{L}} \cdot \boldsymbol{L}_{T}$ & Cost of labor in traditional sectors \\
$\boldsymbol{r}_{T} \cdot \boldsymbol{K}_{T}$ & Cost of capital in traditional sectors \\
$\boldsymbol{w}_{\boldsymbol{L}} \cdot \boldsymbol{L}_{\boldsymbol{R}}$ & Cost of labor in the sectors with robots \\
$\boldsymbol{r}_{\boldsymbol{R}} \cdot \boldsymbol{K}_{\boldsymbol{R}}$ & Cost of capital in the sectors with robots \\
$\boldsymbol{c}_{\boldsymbol{f}}+\boldsymbol{c}_{v} \cdot \boldsymbol{R}$ & Cost of robot in the sectors with robots \\
\hline
\end{tabular}

The investment of the robot requires a certain cost. Thus, $c_{f}$ indicates the fixed cost to be paid by using robots, such as the previous R\&D investment fee and the patent access fee. $c_{v}$ indicates the variable cost to be paid by using robots. Therefore, the cost incurred by enterprises in investing in robots is $c_{f}+c_{v} \cdot R$. Therefore, the profit function of an enterprise can be expressed as ${ }^{1}$ :

$\pi=\left(\theta Y_{T}^{\frac{\sigma-1}{\sigma}}+(1-\theta) Y_{R} \frac{\sigma-1}{\sigma}\right)^{\frac{\sigma}{\sigma-1}}-w_{L T} \cdot L_{T}-r_{T} \cdot K_{T}-w_{L R} \cdot L_{R}-r_{R} \cdot K_{R}-$
$\left(c_{f}+c_{v} \cdot R\right)$.

\section{Analysis of the Optimal Investment Level of Robots}

Since the profit function is complicated (see Appendix 1), without loss of generality, this paper considers the following representative cases when analysing the relationship between the profit of the enterprise and the quantity of the robot input. The substitution elasticity of the production function, $\sigma$, is 1 , and that is, the Cobb-Douglas form is $Y=Y_{R}{ }^{\theta} Y_{T}{ }^{1-\theta}$. The specific output function is $Y=Y_{R}{ }^{\theta} Y_{T}{ }^{1-\theta}=\left(L_{T}^{1-\beta} K_{T}^{\beta}\right)^{\theta}\left(\left(L_{R}+R\right)^{1-\alpha} K_{R}^{\alpha}\right)^{1-\theta}$.

(8)

Now the profit of the firm is

$\pi=\left(L_{T}^{1-\beta} K_{T}^{\beta}\right)^{\theta}\left(\left(L_{R}+R\right)^{1-\alpha} K_{R}^{\alpha}\right)^{1-\theta}-w_{L T} \cdot L_{T}-r_{T} \cdot K_{T}-w_{L R} \cdot L_{R}-r_{R} \cdot K_{R}-c_{f}-$ $c_{v} R$,

(9)

where $w_{L T}=\partial \pi / \partial L_{T}, w_{L R}=\partial \pi / \partial L_{R}, r_{R}=\partial \pi / \partial K_{R}$ and $r_{T}=\partial \pi / \partial K_{T}$.

${ }^{1}$ The expression is shown in Appendix 1

DOI: $10.24818 / 18423264 / 54.2 .20 .12$ 
How Many Robots Should We Invest? A Theoretical Analysis with Two-Sector Production Facility

To simplify the notation, we denote $\lambda=\theta(1-\beta), \mu=(1-\theta)(1-\alpha), \delta=\theta \beta$. Thus, $1-\lambda-\mu-\delta=\alpha(1-\theta)$. Therefore, the profit function can be rewritten as follows:

$\pi=\left(L_{T}^{1-\beta} K_{T}^{\beta}\right)^{\theta}\left(\left(L_{R}+R\right)^{1-\alpha} K_{R}^{\alpha}\right)^{1-\theta}-w_{L T} \cdot L_{T}-r_{T} \cdot K_{T}-w_{L R} \cdot L_{R}-r_{R} \cdot K_{R}-c_{f}-$ $c_{v} R$.

(10)

Substituting $w_{L T}, w_{L R}, r_{R}, r_{T}$ into the profit function, we get the profit function as 2:

$\pi=\mu L_{T}^{\lambda}\left(L_{R}+R\right)^{\mu-1} K_{T}^{\delta} K_{R}^{1-\lambda-\mu-\delta} R-c_{f}-c_{v} R$.

(11)

By solving the optimal number of robot inputs, we have Remark 1 as follows.

Remark 1. When the production function substitution elasticity is 1 , the enterprise has unique optimal investment level of robots $R^{*}$, which is a decreasing function in $c_{v}$, an increasing function in $K_{T}$, and a strictly concave function in $L_{R}$.

Proof: Taking the first-order derivative of $\pi$ with respect to $R$, we have

$\frac{\partial \pi}{\partial R}=\frac{K_{R}{ }^{-\delta-\lambda-\mu}\left(K_{R} K_{T}{ }^{\delta} L_{T}{ }^{\lambda}\left(L_{R}+R\right)^{\mu} \mu\left(L_{R}+R \mu\right)-K_{R}{ }^{\delta+\lambda+\mu}\left(L_{R}+R\right)^{2} c_{v}\right)}{\left(L_{R}+R\right)^{2}}$.

Then we take the second-order derivative of $\pi$ with respect to $R$,

$\frac{\partial^{2} \pi}{\partial R^{2}}=K_{R}{ }^{1-\delta-\lambda-\mu} K_{T}{ }^{\delta} L_{T}^{\lambda}\left(L_{R}+R\right)^{-3+\mu}(-1+\mu) \mu\left(2 L_{R}+R \mu\right)<0$.

According to the second-order derivative above, we show that the profit function has a unique maximizer $R^{*}$, which can be obtained with first-order condition $\partial \pi / \partial R=0$. Defining $F=\partial \pi / \partial R$, the relationship between the optimal investment level and the unit cost can be obtained by using the implicit function derivation method as follows:

$\frac{\partial R^{*}}{\partial c_{v}}=-\frac{F_{\mathcal{C}_{v}}}{F_{R}}=-\frac{-1}{{K_{R}}^{1-\delta-\lambda-\mu_{K_{T}}{ }^{{ }} L_{T}{ }^{\lambda}\left(L_{R}+R\right)^{-3+\mu}(-1+\mu) \mu\left(2 L_{R}+R \mu\right)}}<0$.

The relationship between the optimal investment level of robots and parameters $K_{T}, K_{R}, L_{T}, L_{R}$ can be further obtained in a similar way:

$\frac{\partial R^{*}}{\partial K_{T}}=-\frac{F_{K_{T}}}{F_{R}}=\frac{\left(L_{R}+R\right) \delta\left(L_{R}+R \mu\right)}{K_{T}(1-\mu)\left(2 L_{R}+R \mu\right)}>0$,

$\frac{\partial R^{*}}{\partial K_{R}}=-\frac{F_{K_{R}}}{F_{R}}=\frac{\left(L_{R}+R\right)(-1+\delta+\lambda+\mu)\left(L_{R}+R \mu\right)}{K_{R}(-1+\mu)\left(2 L_{R}+R \mu\right)}>0$,

2 The computation process is illustrated in Appendix 2.

DOI: 10.24818/18423264/54.2.20.12 
Jun Liu, Long Ren, Tong Li

$\frac{\partial R^{*}}{\partial L_{T}}=-\frac{F_{L_{T}}}{F_{R}}=\frac{\left(L_{R}+R\right) \lambda\left(L_{R}+R \mu\right)}{L_{T}(1-\mu)\left(2 L_{R}+R \mu\right)}>0$,

$\frac{\partial R^{*}}{\partial L_{R}}=-\frac{F_{L_{R}}}{F_{R}}=\frac{-R(\mu-1)-L_{R}}{R \mu+2 L_{R}}$,

$\frac{\partial^{2} R^{*}}{\partial L_{R}{ }^{2}}=\frac{(-2+\mu)\left(\mu R^{2}+L R(1+\mu)+L^{2}\right)}{(2 L+\mu R)^{2}\left(R \mu+2 L_{R}\right)}<0$.

Whether $\frac{\partial \mathrm{R}^{*}}{\partial L_{R}}$ is positive or negative depends on $R$ and $L_{R}$. When $R(1-\mu)>L_{R}$, $\frac{\partial \mathrm{R}^{*}}{\partial L_{R}}>0$; when $R(1-\mu)<L_{R}, \frac{\partial \mathrm{R}^{*}}{\partial L_{R}}<0$.

We summarize the comparative statics above in Table 2:

Table 2. Comparative Statics

\begin{tabular}{cccccccc}
\hline & $\boldsymbol{c}_{\boldsymbol{v}}$ & $\boldsymbol{K}_{\boldsymbol{T}}$ & $\boldsymbol{K}_{\boldsymbol{R}}$ & $\boldsymbol{L}_{\boldsymbol{T}}$ & $\boldsymbol{L}_{\boldsymbol{R}}$ \\
\hline Relationship with $\boldsymbol{R}^{*}$ & - & + & + & + & $R(1-\mu)>L_{R},+;$ & $R(1-\mu)<$ \\
& & & & & \\
$L_{R},-$ &
\end{tabular}

In this part, we find that the optimal number of robots that maximize the profit of enterprises and further analyses the factors influencing the optimal number of robots through sensitivity analysis. The main conclusions are as follows. First, the optimal number of robots is negatively correlated with the cost of the robot. As the cost of the robot increases, the optimal investment level of robots will decrease. This is because as the unit cost increases, the risk of investing in robots increases, so the willingness to invest in enterprises decreases. Second, the optimal number of robots is positively correlated with capital. As the capital increases, the more resources will be available to the enterprise, and the more the optimal number of robots will be. Third, the optimal number of robots is positively related to the number of the labor force in the traditional sector. The second and third conclusions are due to the optimal ratio between the output of the firm and the inputs of the traditional sector. As other inputs increase, the number of optimal robots increases. Fourthly, the relationship between the number of optimal robots and the labor force of the robot sector depends on the number of robots and the number of labors in the robot sector. When the number of robots in a certain proportion is greater than the number of labors in the robot sector, the optimal number of robots is positively correlated with the number of labors. When the number of robots in a certain proportion is less than the labor force in the robot sector, the optimal number of robots is negatively correlated with the number of labors. This is because there is a certain substitution relationship between the robot and the labor force of the robot sector. When the labor force is large, it is necessary

DOI: $10.24818 / 18423264 / 54.2 .20 .12$ 
How Many Robots Should We Invest? A Theoretical Analysis with Two-Sector Production Facility

to increase the robot input to achieve the optimal output. The following analyzes the impact of robot inputs on labor wages in different sectors from the perspective of the labor market.

\section{The impact of robot inputs on labor wages}

Section 4 studies the number of robot inputs that maximize the profit of the company from the perspective of enterprise managers. In this section, we will analyse the relationship between the number of robot inputs and labor wages. The general form of the total production function is

$Y=\left(\theta Y_{T}^{\frac{\sigma-1}{\sigma}}+(1-\theta) Y_{R}^{\frac{\sigma-1}{\sigma}}\right)^{\frac{\sigma}{\sigma-1}}=Y=\left(\theta Y_{T}^{\frac{\sigma-1}{\sigma}}+(1-\theta) Y_{R}^{\frac{\sigma-1}{\sigma}}\right)^{\frac{\sigma}{\sigma-1}}$.

From the perspective of enterprises, the wage of the labor force is equal to its marginal income. To that end, the wages of the labor in the traditional sector $w_{L T}$ and the wages in the robot sectors $w_{L R}$ are

$w_{L T}=\frac{\partial Y}{\partial L_{T}}=\theta(1-\beta)\left(L_{T}^{1-\beta} K_{T}^{\beta}\right)^{\frac{\sigma-1}{\sigma}} L_{T}^{-1}\left(\theta\left(L_{T}^{1-\beta} K_{T}^{\beta}\right)^{\frac{\sigma-1}{\sigma}}+(1-\theta)\left(\left(L_{R}+\right.\right.\right.$

$\left.\left.R)^{1-\alpha} K_{R}^{\alpha}\right)^{\frac{\sigma-1}{\sigma}}\right)^{\frac{1}{\sigma-1}}$

$w_{L R}=\frac{\partial Y_{R}}{\partial L_{R}}=(1-\theta)(1-\alpha)\left(\left(L_{R}+R\right)^{1-\alpha} K_{R}^{\alpha}\right)^{\frac{\sigma-1}{\sigma}}\left(L_{R}+R\right)^{-1}\left(\theta\left(L_{T}^{1-\beta} K_{T}^{\beta}\right)^{\frac{\sigma-1}{\sigma}}+\right.$

$\left.(1-\theta)\left(\left(L_{R}+R\right)^{1-\alpha} K_{R}^{\alpha}\right)^{\frac{\sigma-1}{\sigma}}\right)^{\frac{1}{\sigma-1}}$

We then analyse the impacts of the number of robot inputs on labor wages.

Remark 2.As the number of robot inputs increases, the wages of labor in the traditional sectors increases, and the wages in robot sectors decreases.

Proof: Taking the first-order derivative of $w_{L T}$ in $R$, we obtain that the first-order derivative is greater than zero, that is

$$
\begin{aligned}
& \frac{\partial w_{L T}}{\partial R}=\left(L_{R}+R\right)^{-1}(\alpha-1)(\beta-1)(1-\theta) \theta \sigma^{-1} L_{T}^{-1}\left(\left(L_{R}+\right.\right. \\
& \left.R)^{1-\alpha} K_{R}^{\alpha}\right)^{\frac{\sigma-1}{\sigma}}\left(L_{T}^{1-\beta} K_{T}^{\beta}\right)^{\frac{\sigma-1}{\sigma}}\left(\theta\left(L_{T}^{1-\beta} K_{T}^{\beta}\right)^{\frac{\sigma-1}{\sigma}}+(1-\theta)\left(\left(L_{R}+\right.\right.\right. \\
& \left.\left.R)^{1-\alpha} K_{R}^{\alpha}\right)^{\frac{\sigma-1}{\sigma}}\right)^{-1+\frac{1}{\sigma-1}}>0 .
\end{aligned}
$$


Jun Liu, Long Ren, Tong Li

Taking the first-order derivative of $w_{R T}$ in $R$, we have

$\partial w_{L R} / \partial R=\left((1-\alpha)(1-\theta)\left(L_{R}+R\right)^{1-\alpha} K_{R}^{\alpha}\right)^{\frac{\sigma-1}{\sigma}}(\alpha(\theta-$

1) $\sigma K_{R}^{\alpha} L_{T}^{\beta}\left(L_{T}^{1-\beta} K_{T}^{\beta}\right)^{\frac{1}{\sigma}}\left(L_{R}+R\right)-\theta(1+\alpha(\sigma-1)) K_{T}^{\beta} L_{T}\left(L_{R}+R\right)^{\alpha}\left(L_{R}+\right.$

$\left.\left.\left.R)^{1-\alpha} K_{R}^{\alpha}\right)^{\frac{1}{\sigma}}\right)\left(\theta\left(L_{T}^{1-\beta} K_{T}^{\beta}\right)^{\frac{\sigma-1}{\sigma}}+(1-\theta)\left(L_{R}+R\right)^{1-\alpha} K_{R}^{\alpha}\right)^{\wedge}((\sigma-1) / \sigma)\right)^{\wedge}(1 /$

$(\sigma-1))) /\left(\sigma\left(L_{R}+R\right)^{\wedge} 2\left((1-\vartheta) K_{R}^{\alpha} L_{T}^{\beta}\left(L_{T}^{1-\beta} L_{T}^{\beta}\right)^{\wedge}(1 / \sigma)\left(L_{R}+R\right)\right)+\right.$ $\left.\left.\theta K_{T}^{\beta} L_{T}\left(L_{R}+R\right)^{\wedge} \alpha\left(L_{R}+R\right)^{\wedge}(1-\alpha) K_{R}^{\alpha}\right)^{\wedge}(1 / \sigma)\right)$.

The monotonicity of this formula depends on $\alpha(\theta-1) \sigma K_{R}^{\alpha} L_{T}^{\beta}\left(L_{T}^{1-\beta} K_{T}^{\beta}\right)^{\frac{1}{\sigma}}\left(L_{R}+R\right)-$ $\theta(1+\alpha(\sigma-1)) K_{T}^{\beta} L_{T}\left(L_{R}+R\right)^{\alpha}\left(\left(L_{R}+R\right)^{1-\alpha} K_{R}^{\alpha}\right)^{\frac{1}{\sigma}}$. Concerning value range of the parameter, we have $\alpha(\theta-1)<0,-\theta(1+\alpha(\sigma-1))<0$, and thus $\partial w_{L R} / \partial R<0$. The proof is completed.

Furthermore, we analyse the impacts of the number of robot inputs on the ratio of labor wages in these two sectors. The ratio of labor wages in the robot sector and the traditional sector is

$\frac{w_{L R}}{w_{L T}}=\frac{(1-\alpha)(1-\theta)\left(\left(L_{R}+R\right)^{1-\alpha} K_{R}^{\alpha}\right)^{\frac{\sigma-1}{\sigma}} L_{T}}{(1-\beta) \theta\left(L_{T}^{1-\beta} K_{T}^{\beta}\right)^{\frac{\sigma-1}{\sigma}}\left(L_{R}+R\right)}$.

Thus, we have Remark 3 as follows:

Remark 3. The ratio of labor wages in the robot sector and the traditional sector is a decreasing convex function in the number of machine inputs.

Proof:

$\frac{\partial \frac{w_{L R}}{w_{L T}}}{\partial R}=\frac{\frac{(1-\alpha)(\sigma-1)-\sigma}{\sigma}(1-\alpha)(1-\theta) K_{R}^{\frac{\alpha(\sigma-1)}{\sigma}}\left(L_{R}+R\right)^{\frac{(1-\alpha)(\sigma-1)-2 \sigma}{\sigma}}}{(1-\beta) \theta\left(L_{T}^{1-\beta} K_{T}^{\beta}\right)^{\frac{\sigma-1}{\sigma}}}<0$.

This paper will further analyze the second-order derivative of the labor wage ratio of the robot sector and the traditional sector on the number of robots:

$\frac{\partial^{2} \frac{w_{L R}}{w_{L T}}}{\partial R^{2}}=\frac{\frac{(1-\alpha)(\sigma-1)-\sigma(1-\alpha)(\sigma-1)-2 \sigma}{\sigma}(1-\alpha)(1-\theta) K_{R}^{\frac{\alpha(\sigma-1)}{\sigma}}\left(L_{R}+R\right)^{\frac{(1-\alpha)(\sigma-1)-3 \sigma}{\sigma}}}{(1-\beta) \theta\left(L_{T}^{1-\beta} K_{T}^{\beta}\right)^{\frac{\sigma-1}{\sigma}}}>0$. 
How Many Robots Should We Invest? A Theoretical Analysis with Two-Sector Production Facility

The proof is completed.

From the above two remarks, the large-scale application of robots will have a huge impact on the labor wages of the two sectors. On the one hand, as the number of robots increases, the labor force of the robotics sector will gradually decline, while at the same time, the large investment in robots will bring about an increase in labor wages in the traditional sector. The reason for this result is the substitution effect of labor and robots in the robotics sector. The emergence of robots will aggravate the wage gap between the two sectors to a certain extent. From the employee's point of view, the investment of robots will inevitably affect the employment decision-making behavior of the labor force, such as its education level, professional and other relevant decisions; from the perspective of enterprises, the work gap between the two sectors of labor has led to the emergence of coordination problems and increased the difficulty of operation management. From the perspective of society as a whole, the widening wage gap will aggravate social inequality.

\section{Numerical Studies}

This section employs numerical experiments to reveal more economic implications of our theoretical models more intuitively. The basic parameters of numerical studies are as follows:

Table 3. Parameters of Numerical Studies

\begin{tabular}{cccccccccc}
\hline Parameters & $\boldsymbol{c}_{\boldsymbol{v}}$ & $\boldsymbol{K}_{\boldsymbol{T}}$ & $\boldsymbol{K}_{\boldsymbol{R}}$ & $\boldsymbol{L}_{\boldsymbol{T}}$ & $\boldsymbol{L}_{\boldsymbol{R}}$ & $\boldsymbol{\beta}$ & $\boldsymbol{\alpha}$ & $\boldsymbol{\theta}$ & $\boldsymbol{\sigma}$ \\
\hline Values & 1 & 100 & 100 & 100 & 100 & 0.10 & 0.25 & 0.33 & 0.50 \\
\hline
\end{tabular}

Figure 1. The relationship between the optimal number of inputs of the robot and parameters
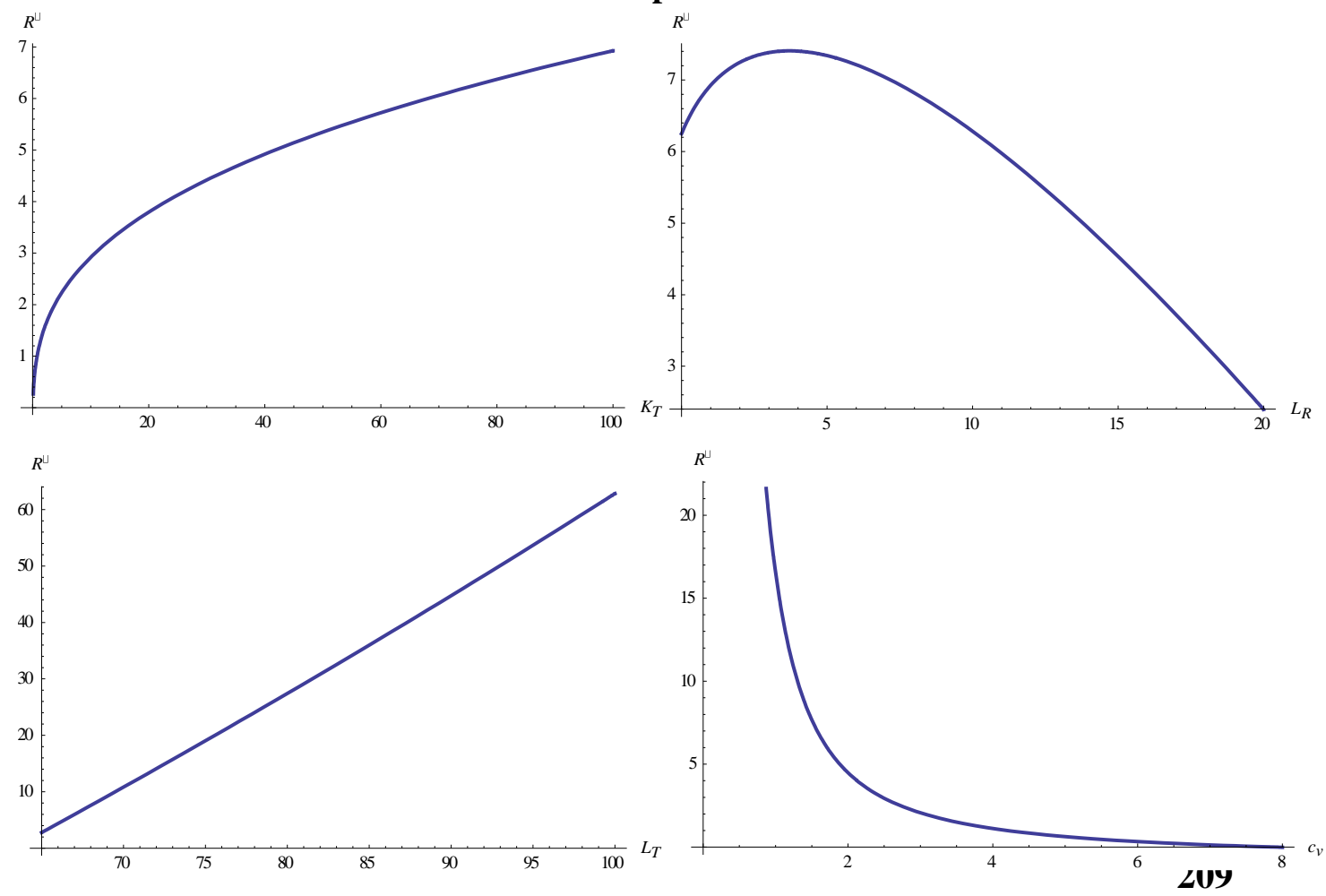

DOI: $10.24818 / 18423264 / 54.2 .20 .12$ 
Jun Liu, Long Ren, Tong Li

Figure 1 shows the relationship between the optimal robot level and parameters. Among them, the optimal investment level is an increasing function in the capital stock $\boldsymbol{K}_{T}$, a decreasing function in the unit production cost $\boldsymbol{c}_{\boldsymbol{v}}$, an increasing function in $\boldsymbol{L}_{T}$, and a firstly increasing and then decreasing function in $\boldsymbol{L}_{\boldsymbol{R}}$, which verifies Section 4 . Further, we verify the relationship between labor wages and various parameters in the robot sector as shown in Figure 2:

Figure 2. The relationship between labor wages and robot input in different sectors
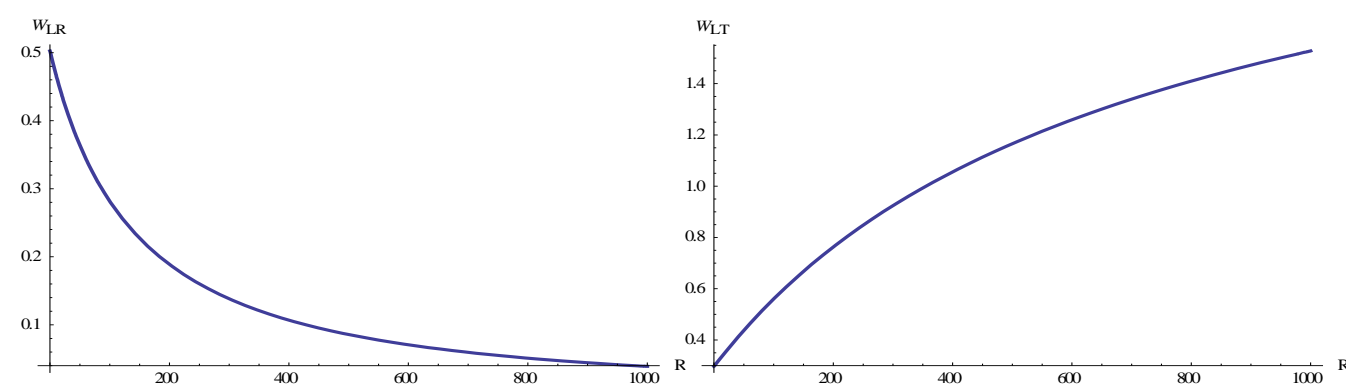

By further analyzing the relationship between labor wages $\left(w_{L R}\right.$ and $\left.w_{L T}\right)$ and the number of robot inputs in each sector, as shown in Figure 2, we find that the wages of labor in the traditional sector increase as the number of robot inputs increases. However, at the same time, the wages of labor in the robot sector decline. As the number of robots increases, the labor force in the robot sector will be gradually replaced by cheaper robotic productivity, resulting in a sharp drop in wages in this sector, while the wages in the traditional sector increases due to its irreplaceability of robots.

Figure 3. The relationship between wages in different sectors and the number of robot inputs

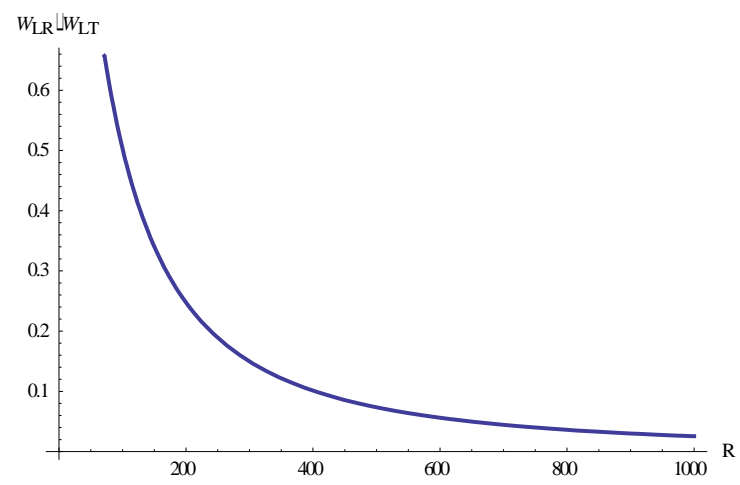

Further, we verify the relationship between the ratio of wages in the robot department against the wages of the traditional department $\left(w_{L R} / w_{L T}\right)$ and the number of robot inputs. As shown in Figure 3, we can observe that as the number of robot inputs increases, the wage ratio of the two departments gradually declines, 
How Many Robots Should We Invest? A Theoretical Analysis with Two-Sector Production Facility

meaning that the wage gap increases. Moreover, the rate of declining decreases as the number of inputs increases. This paper further verifies the relationship between the wage gap in the two sectors and the number of robot inputs through numerical studies.

\section{Conclusions}

With the popularization of robots and artificial intelligence technology, more and more low-cost robots have been extensively used in the production process of enterprises. While robots bring high-efficiency output to enterprises, they will gradually replace the labor force of the enterprise and have an impact on the job market. This paper establishes a two-sector production model by introducing the new production factors of robots. It investigates the driven forces of the optimal number of robots from the perspective of enterprise managers, and the relationship between robot inputs and the profits of enterprises, and then discusses the relationship between the number of robots and the wages of labor from a perspective of the labor market.

Through the analysis, we find that the optimal investment level of the robot depends mainly on the unit cost of the robot, the capital stock, and the number of human workforces in these two sectors. A higher unit cost of the robot leads to a decreasing investment level of robots. The higher the capital stock of the traditional sector and the higher number of workforces in the traditional sector, the higher investment level of robots. From the perspective of workforce wages, the increase in the number of robot inputs will cause a deepened wage gap between the two sectors. In addition, from a viewpoint of the social planner, in order to solve the social injustice caused by the robot inputs, the inequality can be reduced by taxing the robot or transferring payment between these two sectors. Thus, the role of the social planner departments is predominant. For policymakers, on one hand, a more rational distribution mechanism should be established. On the other hand, vocational education can be employed to transform labor in traditional sectors to sectors that cannot be replaced by robots. The laborers should engage in activities where they have comparative advantages to prevent the fairness problem caused by ongoing robot investments.

There are some research potentials that can be extended along with this paper. With the development of artificial intelligence technology, can we achieve the true "strong artificial intelligence", that is, when considering the variable cost of the robot as a function of the reduction of the investment level, how will the impact of the "strong artificial intelligence" of economic development on the wages of the labor market change?

\section{REFERENCES}

[1]Acemoglu, D. \& Autor, D. (2011),Skills, Tasks and Technologies: Implications for Employment and Earnings. Handbook of labor economics, 4, 1043-1171;

DOI: $10.24818 / 18423264 / 54.2 .20 .12$ 
Jun Liu, Long Ren, Tong Li

[2]Acemoglu, D. \& Restrepo, P. (2017), Secular Stagnation? The Effect of Aging on Economic Growth in the Age of Automation.American Economic Review, 107(5), 174-79;

[3]Acemoglu, D. \& Restrepo, P. (2018), The Race between Man and Machine: Implications of Technology for Growth, Factor Shares and Employment.American Economic Review, 108(6), 1488-1542;

[4]Autor, D. H., Katz, L. F. \& Krueger, A. B. (1998), Computing Inequality: Have Computers Changed the Labor Market?.The Quarterly journal of economics, 113(4), 1169-1213;

[5]Autor, D.\&Salomons, A. (2018), Is Automation Labor Share-Displacing? Productivity Growth, Employment, and the Labor Share.Brookings Papers on Economic Activity, 2018(1), 1-87;

[6]Benzell, S. G., Kotlikoff, L. J., LaGarda, G. \& Sachs, J. D. (2015), Robots Are Us: Some Economics of Human Replacement. National Bureau of Economic Research. No. w20941;

[7]Carneiro, P. \& Lee, S. (2011),Trends in Quality-adjusted Skill Premia in the United States, 1960-2000. American Economic Review, 101(6), 2309-49;

[8]Diamond, P. A. (1965),National Debt in a Neoclassical Growth Model. The American Economic Review, 55(5), 1126-1150;

[9]Frey, C. B. \& Osborne, M. A. (2017), The Future of Employment: How Susceptible Are Jobs to Computerisation?. Technological forecasting and social change, 114, 254-280;

[10]Gasteiger, E. \& Prettner, K. (2017), On the Possibility of Automation-Induced Stagnation. Hohenheim Discussion Papers in Business, Economics and Social Sciences. No. 07-2017;

[11]Gong, D., Liu, S., Liu, J. \& Ren, L. (2019), Who Benefits from Online Financing? A Sharing Economy E-Tailing Platform Perspective. International Journal of Production Economics;

[12]Graetz, G. \& Michaels, G. (2018), Robots at Work. Review of Economics and Statistics, 100(5), 753-768;

[13]Katz, L. F. \& Murphy, K. M. (1992), Changes in Relative Wages, 1963-1987: Supply and Demand Factors. The quarterly journal of economics, 107(1), 35-78;

[14]Kromann, L., Skaksen, J. R. \&Sørensen, A. (2011), Automation, Labor Productivity and Employment-A Cross Country Comparison.CEBR, Copenhagen Business School;

[15]Prettner, K. \& Strulik, H. (2017),The Lost Race against the Machine: Automation, Education, and Inequality in an R\&D-Based Growth Model. Cege Discussion Papers;

[16]Prettner, K. (2017), A Note on the Implications of Automation for Economic Growth and the Labor Share. Macroeconomic Dynamics, 1-8;

[17]Sachs, J. D. \& Kotlikoff, L. J. (2012),Smart Machines and Long-term Misery. National Bureau of Economic Research. No. w18629;

DOI: $10.24818 / 18423264 / 54.2 .20 .12$ 
How Many Robots Should We Invest? A Theoretical Analysis with Two-Sector Production Facility

[18]Solow, R. M. (1956), A Contribution to the Theory of Economic Growth. The quarterly journal of economics, 70(1), 65-94;

[19]Su, C. W., Li, Z. Z., Chang, H. L. \& Lobonţ, O. R. (2017), When Will Occur the Crude Oil Bubbles?. Energy Policy, 102, 1-6;

[20]Su, C. W., Wang, X. Q., Tao, R. \& Lobont O.R. (2019),Do Oil Prices Drive Agricultural Commodity Prices? Further Evidence in a Global Bio-energy Context. Energy, 172, 691-701;

[21]Tinbergen, J. (1974),Substitution of Graduate by other Labour. Kyklos: international review for social sciences;

[22]Tinbergen, J. (1975),Income Differences: Recent Research;

[23]Zeira, J. (1998), Workers, Machines, and Economic Growth. The Quarterly Journal of Economics, 113(4), 1091-1117.

\section{Appendix 1}

The expression of profit function is

$$
\begin{aligned}
& \pi=\left(\theta Y_{T}^{\frac{\sigma-1}{\sigma}}+(1-\theta) Y_{R}^{\frac{\sigma-1}{\sigma}}\right)^{\frac{\sigma}{\sigma-1}}-\left[\theta ( 1 - \beta ) ( L _ { T } ^ { 1 - \beta } K _ { T } ^ { \beta } ) ^ { \frac { \sigma - 1 } { \sigma } } L _ { T } ^ { - 1 } \left(\theta\left(L_{T}^{1-\beta} K_{T}^{\beta}\right)^{\frac{\sigma-1}{\sigma}}+\right.\right. \\
& \left.\left.(1-\theta)\left(\left(L_{R}+R\right)^{1-\alpha} K_{R}^{\alpha}\right)^{\frac{\sigma-1}{\sigma}}\right)^{\frac{1}{\sigma-1}}\right] \cdot L_{T}-\left[\theta \beta ( L _ { T } ^ { 1 - \beta } K _ { T } ^ { \beta } ) ^ { \frac { \sigma - 1 } { \sigma } } K _ { T } ^ { - 1 } \left(\theta\left(L_{T}^{1-\beta} K_{T}^{\beta}\right)^{\frac{\sigma-1}{\sigma}}+\right.\right. \\
& \left.\left.(1-\theta)\left(\left(L_{R}+R\right)^{1-\alpha} K_{R}^{\alpha}\right)^{\frac{\sigma-1}{\sigma}}\right)^{\frac{1}{\sigma-1}}\right] \cdot K_{T}-\left[( 1 - \theta ) ( 1 - \alpha ) ( ( L _ { R } + R ) ^ { 1 - \alpha } K _ { R } ^ { \alpha } ) ^ { \frac { \sigma - 1 } { \sigma } } \left(L_{R}+\right.\right. \\
& \left.R)^{-1}\left(\theta\left(L_{T}^{1-\beta} K_{T}^{\beta}\right)^{\frac{\sigma-1}{\sigma}}+(1-\theta)\left(\left(L_{R}+R\right)^{1-\alpha} K_{R}^{\alpha}\right)^{\frac{\sigma-1}{\sigma}}\right)^{\frac{1}{\sigma-1}}\right] \cdot L_{R}-\left[( 1 - \theta ) \alpha \left(\left(L_{R}+\right.\right.\right. \\
& \left.\left.R)^{1-\alpha} K_{R}^{\alpha}\right)^{\frac{\sigma-1}{\sigma}} K_{R}^{-1}\left(\theta\left(L_{T}^{1-\beta} K_{T}^{\beta}\right)^{\frac{\sigma-1}{\sigma}}+(1-\theta)\left(\left(L_{R}+R\right)^{1-\alpha} K_{R}^{\alpha}\right)^{\frac{\sigma-1}{\sigma}}\right)^{\frac{1}{\sigma-1}}\right] \cdot K_{R}- \\
& \left(c_{f}+c_{v} \cdot R\right) . \\
& (\mathrm{A} 1)
\end{aligned}
$$

\section{Appendix 2}

The computation process of enterprise profits is $\pi=L_{T}{ }^{\lambda}\left(L_{R}+R\right)^{\mu} K_{T}^{\delta} K_{R}^{1-\lambda-\mu-\delta}-w_{L T} \cdot L_{T}-r_{T} \cdot K_{T}-w_{L R} \cdot L_{R}-r_{R} \cdot K_{R}-c_{f}-c_{v} R$ 
Jun Liu, Long Ren, Tong Li

$$
\begin{aligned}
& =L_{T}{ }^{\lambda}\left(L_{R}+R\right)^{\mu} K_{T}^{\delta} K_{R}^{1-\lambda-\mu-\delta}-\lambda{L_{T}}^{\lambda}\left(L_{R}+R\right)^{\mu} K_{T}^{\delta} K_{R}^{1-\lambda-\mu-\delta}-\mu{L_{T}}^{\lambda}\left(L_{R}+\right. \\
& R)^{\mu} K_{T}^{\delta} K_{R}^{1-\lambda-\mu-\delta} \frac{L_{R}}{L_{R}+R}-\delta L_{T}^{\lambda}\left(L_{R}+R\right)^{\mu} K_{T}^{\delta} K_{R}^{1-\lambda-\mu-\delta}-(1-\lambda-\mu-\delta) L_{T}^{\lambda}\left(L_{R}+\right. \\
& R)^{\mu} K_{T}^{\delta} K_{R}^{1-\lambda-\mu-\delta}-c_{f}-c_{v} R=\mu L_{T}^{\lambda}\left(L_{R}+R\right)^{\mu-1} K_{T}^{\delta} K_{R}^{1-\lambda-\mu-\delta} R-c_{f}-c_{v} R .(\mathrm{A} 2)
\end{aligned}
$$

